\title{
artigo
}

\section{Efeitos e segurança do uso off label do topiramato na perda de peso}

\author{
Effects and safety of use off label of the topiramate on weight loss \\ Efectos y seguridad de uso off label del topiramato en la pérdida de peso
}

\begin{abstract}
RESUMO
Objetivo: realizar um levantamento na literatura nacional e internacional sobre a eficácia e a segurança do Topiramato no uso 0 -label na perda de peso. Método: Trata-se de uma revisão integrativa, na qual a busca dos dados, foi realizada no PUBMED, Embase e Cochrane, entre os anos de 2016 a 2021, foram selecionadas 10 pesquisas. Resultados: o Topiramato pode ser uma ferramenta terapêutica segura e eficaz no tratamento da obesidade, proporcionando redução do índice de massa corpórea, favorecendo o controle glicêmico e a pressão arterial, tanto em adultos, quanto em adolescentes. Os efeitos adversos associados ao tratamento incluíram principalmente disgeusia, parestesia e boca seca. Conclusão: o Topiramato utilizado de forma isolada ou em combinação com a metformina, a fentermina, bupropiona-naltrexona e a Ispagula mostrou efeitos vantajosos na perda de peso.
\end{abstract}

DESCRITORES: Topiramato; Tratamento; Obesidade. Efeitos e segurança; Fármacos para Perda de Peso.

\section{ABSTRACT}

Objective: to conduct a survey in the national and international literature on the efficacy and safety of Topiramate in o -label use in weight loss. Method: This is an integrative review, in which the search for data was carried out in PUBMED, Embase and Cochrane, between the years 2016 to 2021, 10 researches were selected. Results: Topiramate can be a safe and effective therapeutic tool in the treatment of obesity, providing a reduction in body mass index, favoring glycemic control and blood pressure, both in adults and adolescents. Adverse effects associated with treatment mainly included dysgeusia, paresthesia and dry mouth. Conclusion: Topiramate used alone or in combination with metformin, phentermine, bupropion-naltrexone and Ispagula showed beneficial effects on weight loss.

DESCRIPTORS: Topiramate; Treatment; Obesity. Effects and safety; Weight Loss Drugs.

\section{RESUMEN}

Objetivo: realizar una encuesta en la literatura nacional e internacional sobre la eficacia y seguridad del topiramato en uso sin receta en la pérdida de peso. Método: Se trata de una revisión integradora, en la que la búsqueda de datos se realizó en PUBMED, Embase y Cochrane, entre los años 2016 a 2021, se seleccionaron 10 investigaciones. Resultados: El topiramato puede ser una herramienta terapéutica segura y eficaz en el tratamiento de la obesidad, proporcionando una reducción del índice de masa corporal, favoreciendo el control glucémico y la presión arterial, tanto en adultos como en adolescentes. Los efectos adversos asociados con el tratamiento incluyeron principalmente disgeusia, parestesia y sequedad de boca. Conclusión: El topiramato usado solo o en combinación con metformina, fentermina, bupropión-naltrexona e Ispagula mostró efectos beneficiosos sobre la pérdida de peso. DESCRIPTORES: topiramato; Tratamiento; Obesidad. Efectos y seguridad; Medicamentos para bajar de peso.

RECEBIDO EM: 17/05/2021 APROVADO EM: 04/06/2021

\section{Ana Flávia Machado de Carvalho}

Fisoterapeuta. Doutora em Engenharia Biomédica. Professora efetiva da Universidade Estadual do Piauí e da UNIFacid/Wyden. Especialização Em Saúde Pública, Universidade Federal Do Piauí, Piauí, Brasil. Ano de obtenção: 2002; Graduação Em Fisioterapia, Universidade Estadual da Paraíba, UEPB, Brasil. Ano de obtenção: 1996.

ORCID: 0000-0002-6691-4804

\section{Fernando Antônio Madeira Marinho}

Médico. Plantonista de SAMU e Mestrando em Biotecnologia e Atenção Básica de Saúde. Especialização em Segurança do Paciente, Fundação Oswaldo Cruz, Ano de Obtenção: 2017; Graduação em Medicina, Centro Universitário Tocantinense Presidente Antônio Carlos, Ano de Obtenção: 2010.

ORCID: 0000-0003-3703-9366 


\section{Francisco Laurindo da Silva}

Orientador. Mestrado em Ciências Biológicas Professor da UNIFacid/Wyden. Doutorado em Ciências Biológicas, Universidade Federal de Minas Gerais, Ano de obtenção: 2004; Mestrado em Ciências Biológicas, Universidade Federal de Minas Gerais, Ano de obtenção: 200; Graduação em Ciências Biológicas, Universidade Federal do Piauí, Ano de obtenção: 1997.

ORCID: 0000-0001-6837-4509

\section{INTRODUÇÃO}

A obesidade é uma doença crônica de origem multifatorial caracterizada pelo excesso de adiposidade corpórea, que ocasiona prejuízos à saúde. A obesidade vem assumindo proporções epidêmicas em quase todos os países ${ }^{1}$. De acordo com projeções da Organização Mundial da Saúde (OMS), estima-se que em torno de 2,3 bilhões de adultos no mundo estejam acima do peso e que 700 milhões desses sejam considerados obe$\operatorname{sos}^{2}$. No Brasil, a proporção de indivíduos obesos em 2017 foi de 18,9\%, com maior representação entre os homens $(19,2 \%)$ do que entre as mulheres $(18,7 \%)^{3-4}$. A obesidade pode ser classificada em endógena e exógena ${ }^{5}$. O tratamento da obesidade abrange abordagens nutricionais, farmacológicas e/ou prática de atividade física, porém essas técnicas e mudanças de estilo de vida têm se mostrado ineficazes (98\% de reincidência) em indivíduos com obesidade mórbida ${ }^{6-8}$.

Para o tratamento farmacológico da obesidade, estudo demostrou como os mais utilizados a Sibutramina (65\%), em seguida o Orlistate (26,25\%), Fluoxetina (3,75\%), Duloxitina (2,5\%) e Topiramato $(2,5 \%)$, lembrando que os três últimos são medicamentos off label (fora da bula) ou seja são comercializados para o tratamento da ansiedade e depressão?

No que diz respeito ao Topiramato, ressalta-se que é uma medicação indicada para o tratamento da convulsão, sendo considerado um monossacarídeo sulfamato substituído. Atualmente tem sido utilizado no manejo de migrânea, enxaqueca, dor neuropática, síndrome depressiva e obesidade. Além disso, o Topiramato vem sendo empregado de forma off label no tratamento da perca de peso. Todavia, ainda é desconhecido seu mecanismo de ação como causa de redutor de peso, sabe-se
A obesidade é uma doença crônica de origem multifatorial

caracterizada

pelo excesso de

adiposidade corpórea, que ocasiona prejuízos

à saúde. A obesidade

vem assumindo

proporçôes

epidêmicas em quase

todos os países. De

acordo com projeções

da Organização

Mundial da Saúde

(OMS), estima-se

que em torno de 2,3

bilhões de adultos no

mundo estejam acima

do peso e que 700

milhões desses sejam

considerados obesos que ele é inibidor de neurotransmissores, como serotonina, noradrenalina fazendo com que ocorra a saciedade ${ }^{9-10}$.

$\mathrm{O}$ uso de medicação off label consiste em tratamento experimental, sem qualquer evidência científica de sua eficácia, o que pode causar riscos à vida dos pacientes $^{10}$. Todavia, o que se percebe pelo contexto da dispensa de tais classes medicamentosas é que a saúde financeira das indústrias farmacológicas é o maior beneficiário da comercialização desses medicamentos ${ }^{11-12}$. Levando em consideração os impactos ocasionados em virtude da obesidade e a diversidade de tratamentos existentes surgiu o interesse em analisar na literatura a eficácia e a segurança do Topiramato no uso off label na pera de peso. Portanto, o objetivo geral desta pesquisa foi realizar um levantamento na literatura nacional e internacional sobre a eficácia e a segurança do Topiramato no uso off label na perda de peso, e especificamente foi intenção, identificar as vantagens e desvantagens do uso Topiramato na perda de peso, demostrar as dosagens e o tempo de uso do Topiramato na perda de peso.

\section{MÉTODOS}

Tratou-se de uma pesquisa de revisão, do tipo integrativa, desenvolvida nas seguintes fases: elaboração da pergunta norteadora busca ou amostragem na literatura, coleta de dados, análise crítica dos estudos incluídos, discussão dos resultados e apresentação da revisão integrativa. A pergunta de pesquisa foi: qual a eficácia e a segurança do Topiramato no uso off label na perda de peso?

A busca por pesquisas foi realizada no PUBMED, Embase e Cochrane, por meio da combinação dos seguintes descritores na língua inglesa: Topiramate; Treatment; Obesity; Effects and safety; Weight Loss Drugs (de acordo com Medical Subject 


\section{artigo}

Carvalho, A.F.M.; Marinho, F.A.M.; Silva, F.L.;

Efeitos e segurança do uso off label do topiramato na perda de peso

Headings - MESH). Esses descritores foram usados nas bases de dados mencionados com o conectivo boleano "and".

Foram incluídas no estudo pesquisas primárias, relatos de experiência publicados no período de janeiro de 2016 a março de 2021, em língua portuguesa, inglesa e espanhol, que estudaram os efeitos do Topiramato na perda de pesos de pacientes obesos. Foram excluídas pesquisas duplicadas, realizadas em animais, que fugiam do objetivo proposto por este estudo e as pesquisas de revisão, com exceção as meta-análises.

Foram identificadas 356 pesquisas $(\mathrm{PUBMED}=156$; Embase $=100$ e Cochrane $=100)$, dos quais 51 deles foram excluídos por se tratar de artigos duplicados, 120 por ser uma pesquisa em animais, 120 artigos por fugir do objetivo proposto, e 55 por se tratar de pesquisas de revisão (integrativa e narrativa), restando hg 10 artigos que foram analisados na integra.

Os ados coletados foram submetidos a uma leitura minuciosa para seleção. As pesquisas foram caracterizadas em quadro síntese e os resultados foram distribuídos de forma discursiva, fazendo uma comparação entre os diferentes achados por meio de uma padronização dos conteúdos, que foram agrupados de acordo com sua similaridade. Por fim os dados foram validados por dois pesquisadores.

\section{RESULTADOS}

Os resultados deste estudo foram construídos por meio da análise e interpretação de 10 pesquisas que respondiam aos obje- tivos propostos, com maior destaque para aquelas publicadas no ano de 2020, com três selecionadas. Quanto a metodologia adotada por esses estudos, destacaram-se os ensaios clínicos. Apenas uma pesquisa avaliou o Topiramato de forma isolada na perda de peso $^{13}$. Duas pesquisas avaliaram a combinação do Topiramato com a metformina. Outras cinco avaliaram a combinação do Topiramato com a fentermina ${ }^{14-20}$. O quadro 1 mostra as bases de dados, o país de origem, o título, o tipo de estudo, os autores, o periódico e ano de publicação.

\section{DISCUSSÃO}

O Topiramato tem um papel estabelecido como anticonvulsivante, mas pode ter um papel no tratamento da obesidade,

\section{Quadro 1: Caracterização dos resultados das pesquisas selecionadas.}

\begin{tabular}{|c|c|c|c|c|c|}
\hline $\begin{array}{l}\text { BASES DE } \\
\text { DADOS }\end{array}$ & $\begin{array}{l}\text { PAÍS DE } \\
\text { ORIGEM }\end{array}$ & TÍTULO & $\begin{array}{l}\text { TIPO DE } \\
\text { ESTUDO }\end{array}$ & AUTORES & $\begin{array}{l}\text { PERIÓDICOS E ANO } \\
\text { DE PUBLICAÇÃO }\end{array}$ \\
\hline PUBMED & $\begin{array}{l}\text { Estados } \\
\text { Unidos da } \\
\text { América }\end{array}$ & $\begin{array}{l}\text { Health-related quality of life in two ran- } \\
\text { domized controlled trials of phentermine/ } \\
\text { topiramate for obesity: What mediates } \\
\text { improvement? }\end{array}$ & $\begin{array}{l}\text { Ensaios clínicos } \\
\text { randomizados }\end{array}$ & Kolotkin et al. & Qual Life Res/ 2016 \\
\hline PUBMED & Brasil & $\begin{array}{l}\text { Safety assessment of combination } \\
\text { therapies in the treatment of obesity: } \\
\text { focus on naltrexone/bupropion extended } \\
\text { release and phentermine-topiramate } \\
\text { extended release }\end{array}$ & Revisão clínica & $\begin{array}{l}\text { Halpern e } \\
\text { Mancini }\end{array}$ & $\begin{array}{l}\text { Expert opin drug } \\
\text { saf./2017 }\end{array}$ \\
\hline Embase & $\begin{array}{l}\text { Estados } \\
\text { Unidos da } \\
\text { América }\end{array}$ & $\begin{array}{c}\text { An Innovative Disease-Drug-Trial Fra- } \\
\text { mework to Guide Binge Eating Disorder } \\
\text { Drug Development: A Case Study for } \\
\text { Topiramato. }\end{array}$ & Estudo de caso & Kalaria et al. & Clin TransI Sci/ 2019 \\
\hline PUBMED & $\begin{array}{l}\text { Estados } \\
\text { Unidos da } \\
\text { América }\end{array}$ & $\begin{array}{l}\text { A randomized, double-blind, placebo-con- } \\
\text { trolled, pharmacokinetic and pharmacody- } \\
\text { namic study of a fixed-dose combination } \\
\text { of phentermine/ topiramate in adoles- } \\
\text { cents with obesity. }\end{array}$ & $\begin{array}{l}\text { Um estudo ran- } \\
\text { domizado, duplo- } \\
\text {-cego, controlado } \\
\text { por placebo }\end{array}$ & Hsia et al. & $\begin{array}{c}\text { Diabetes Obes Metab/ } \\
2020\end{array}$ \\
\hline PUBMED & Israel & $\begin{array}{l}\text { The Mitigating Effect of Phentermine } \\
\text { and Topiramate on Weight Regain After } \\
\text { Roux-en-Y Gastric Bypass Surgery }\end{array}$ & $\begin{array}{l}\text { Ensaio clínico } \\
\text { randomizado }\end{array}$ & Istfan et al. & $\begin{array}{c}\text { Obesity (Silver Spring)/ } \\
2020\end{array}$ \\
\hline Cochrane & China & $\begin{array}{c}\text { Efficacy and safety of phentermine/ } \\
\text { topiramate in adults with overweight or } \\
\text { obesity: A meta-analysis and systematic } \\
\text { review }\end{array}$ & $\begin{array}{l}\text { Uma revisão } \\
\text { sistemática e } \\
\text { meta-análise }\end{array}$ & Lei et al. & $\begin{array}{l}\text { MedRxiv preprint/ } \\
2020\end{array}$ \\
\hline Embase & Israel & $\begin{array}{c}\text { A randomized, placebo controlled crosso- } \\
\text { ver trial of phentermine topiramate ER in } \\
\text { patients with binge eating disorder and } \\
\text { bulimia nervosa. }\end{array}$ & $\begin{array}{l}\text { Ensaio cruzado } \\
\text { randomizado e } \\
\text { controlado por } \\
\quad \text { placebo }\end{array}$ & Safer et al. & First published/ 2020 \\
\hline
\end{tabular}




\begin{tabular}{|c|c|c|c|c|c|}
\hline PUBMED & China & $\begin{array}{c}\text { Outcomes and safety of concomitant } \\
\text { topiramate or metformin for antipsycho- } \\
\text { tics-induced obesity: a randomized-con- } \\
\text { trolled trial. }\end{array}$ & $\begin{array}{c}\text { Ensaio clínico } \\
\text { randomizado }\end{array}$ & Wang et al. & $\begin{array}{c}\text { Ann Gen Psychiatry/ } \\
2020\end{array}$ \\
\hline PUBMED & Índia & $\begin{array}{c}\text { Study on Efficacy of Topiramate: Impact } \\
\text { on weight Loss in Obese patients. }\end{array}$ & $\begin{array}{c}\text { Ensaio clínico } \\
\text { randomizado }\end{array}$ & $\begin{array}{c}\text { Nithyakala e } \\
\text { Sathyaprabha }\end{array}$ & $\begin{array}{c}\text { Expert opin drug saf/ } \\
2021\end{array}$ \\
\hline PUBMED & $\begin{array}{c}\text { Estados } \\
\text { Unidos da } \\
\text { América }\end{array}$ & $\begin{array}{c}\text { Case Report: Off Label Utilization of Topi- } \\
\text { ramate and Metformin in Patients With } \\
\text { BMl } \geq 50 \mathrm{~kg} / \mathrm{m} 2 \text { Prior to Bariatric Surgery. }\end{array}$ & Relato de caso & $\begin{array}{c}\text { Sari, Seip e } \\
\text { Umashanker }\end{array}$ & $\begin{array}{c}\text { Case Report: Pharma- } \\
\text { cological Weight Loss } \\
\text { With Generics 2021 }\end{array}$ \\
\hline Fonte: PUBMED, Embase Cochrane & \multicolumn{2}{|l|}{} & & \\
\hline
\end{tabular}

conforme mostrou a maioria das pesquisas analisadas, pois apenas em uma das pesquisas foi possível identificar, que a terapia combinada de Topiramato e fentermina não foi bem tolerada, em que mais da metade dos pacientes parou de tomá-lo devido aos efeitos adversos ${ }^{16}$.

Duas pesquisas concordam que o uso do Topiramato como primeira linha de terapia para perda de peso é limitada devido ao seu perfil de efeitos colaterais, que inclui disfunção cognitiva, parestesia, perversão do paladar, acidose metabólica e toxicidade ocular podem ocorrer como efeitos adversos. Desta maneira, para a seleção da dose apropriada é necessária para maximizar o benefício de reduzir e limitar os riscos associados a efeitos adversos ${ }^{13,15}$. De forma diferente, um estudo que analisou a combinação do Topiramato com a fentermina na perda de peso de pacientes com bulimia nervosa, identificou que essa combinação se mostrou segura e eficaz, sendo bem tolera$\mathrm{da}$, não sendo mencionadas desvantagen ${ }^{20}$. Em outra pesquisa, o Topiramato foi utilizado em pacientes obesos com transtorno da compulsão alimentar periódica. Já no grupo controle a relação dose-resposta identificada demonstrou que uma dose diária de $125 \mathrm{mg}$ de Topiramato foi necessária para exibir uma redução acentuada na perda de peso semanal ${ }^{13}$.

$\mathrm{Na}$ maioria dos estudos analisados a principal vantagem da utilização do Topiramato, seja ele individualmente ou em combinação com a fentermina ou metformina, foi a perda de peso significativa, a qual variou de 5 a mais de $10 \%$, melhorando os fatores de risco cardiovascular, incluindo redução da pressão arterial sistólica e níveis de glicose $\mathrm{e}^{14,15,18-18}$.
Em um estudo realizado nos Estados Unidos da América, com três pacientes, identificou à associação do Topiramato com a metformina, tendo como vantagens os efeitos de modificar o impulso de fome, incluindo a redução dos desejos e aumento da sensação de saciedade. Os medicamentos podem ser usados sozinhos ou em combinação para facilitar a perda de peso pré-operatória ${ }^{15}$. De forma semelhante ao estudo anterior, autores exploraram a eficácia e segurança do Topiramato e da metformina em pacientes chineses, sendo possível constatar que essas duas medicações surtiram efeitos eficazes na redução da obesidade, reduzindo significativamente o peso corporal, o IMC e a relação cintura-quadril e boa tolerância em pacientes psiquiátricos, não sendo observada nenhuma desvantagem em sua utilização ${ }^{14}$.

Outra pesquisa analisou 1.196 prontuários eletrônicos de pacientes submetidos a cirurgia bariátrica entre 2004 e 2015 e mostrou que a fentermina e o Topiramato, usados individualmente ou em combinação, podem reduzir significativamente a perda de peso após cirurgia bariátrica. Não foram evidenciadas desvantagem da utilização dessas medicações ${ }^{18}$.

Em outro estudo foi possível identificar as mesmas vantagens da combinação destas duas medicações anteriormente mencionadas e trouxeram ainda benefícios diretos a qualidade de vida relacionada a saúde de $88,2 \%$ dos 2.374 pacientes avaliados ${ }^{15}$. Em uma meta-análise de ensaios clínicos randomizados a terapia com fentermina combinada com o Topiramato resultou em uma perda de peso de $7,73 \mathrm{~kg}$, reduziu a circunferência da cintura, a pressão arterial, os níveis de açú- car no sangue e os níveis de lipídios. Os efeitos adversos associados ao tratamento incluíram principalmente disgeusia, parestesia, boca seca ${ }^{19}$.

Em outra meta-análise, as combinação de dose fixa de bupropiona-naltrexona e combinações de dose titulada de fentermina e Topiramato foram avaliadas em 2.410 pacientes, sendo possível identificar eficácia e segurança, com os mesmos efeitos adversos mencionados no estudo anterior ${ }^{21}$. Outros autores avaliaram a combinação de dose fixa de fentermina de liberação imediata e Topiramato de liberação estendida em adolescentes de 12 a 17 anos com obesidade e constataram que à perda de peso foi estatisticamente significativa e o perfil de segurança foi tolerável. Esses dados indicam que os níveis de dose média e alta são apropriados e com eficácia de longo prazo em adolescentes ${ }^{17}$.

Com abordagem diferente dos estudos anteriores, foi testado Topiramato e ispagula em pacientes com obesidade por 6 meses. A dose final de Topiramato $25 \mathrm{mg}$ a $50 \mathrm{mg} /$ por dia e Ispagula $1,2 \mathrm{~g}$ a $3,6 \mathrm{~g}$ / por dia variou, sendo possível identificar que o Topiramato pode ser considerado no tratamento de pacientes com obesidade. Ao comparar os resultados médios de IMC de ispagula e tropiramato, observou-se que o Topiramato é mais eficaz na redução de peso nos pacientes do Grupo 2 (teste) ${ }^{22}$.

\section{CONCLUSÃO}

Constatou-se que o Topiramato pode ser uma ferramenta terapêutica adjuvante eficaz no tratamento da obesidade, desde que os efeitos colaterais sejam considerados. Essa medicação mostrou efeitos sig- 


\section{artigo}

nificativos na perda de peso, na redução do IMC, redução do ganho de peso após a cirurgia bariátrica, no controle glicêmico e da pressão arterial, tanto em adultos, quanto em adolescentes. Essas vantagens foram observadas em sua utilização de forma isolada, como também em combinação com a metformina ou com a fentermina.
No que se refere a dosagem recomendada não foi possível identificar um consenso entre a literatura analisada. Todavia, a dosagem mínima por dia utilizada foi de $50 \mathrm{mg}$ e a máxima foi de $150 \mathrm{mg}$, por um período mínimo de 3 meses.

As desvantagens da utilização do Topiramato no controle da obesidade esteve- ram relacionadas aos efeitos adversos desta medicação, que podem influenciar na tolerância dos pacientes e consequentemente desistência do tratamento. São necessários mais estudos clínicos e de farmacovigilância de longo prazo para compreender a eficácia e segurança de longo prazo do Topiramato.

\section{REFERÊNCIAS}

1. Barros F, Negrão MG, Negrão, GG. Comparação da perda de peso após sleeve e bypass gástrico em y-de-roux: revisão sistemática. ABCD, arq. bras. cir. dig. 2019; 32 (4): 21-30.

2. World Health Organization. Obesity and overweight. 2018. Disponivel em:<https://www.who.int/news-room/fact-sheets/ detail/obesity-and-overweight. Acesso em: 02 mar. 2021.

3. Brasil. Vigitel Brasil: vigilância de fatores de risco e proteção para doenças crônicas por inquérito telefônico: estimativas sobre frequência e distribuição sociodemográfica de fatores de risco e proteção para doenças crônicas nas capitais dos 26 estados brasileiros e no Distrito Federal em 2017. Brasília: Ministério da Saúde; 2018.

4. Lima NMS, Leal VS, Oliveira JS, Siqueira MIA, Lima FCPT, Egito RCM, Silva CS, Cabral PIL. Excesso de peso em adolescentes e estado nutricional dos pais: uma revisão sistemática. Ciênc. saúde coletiva. 2017; 22 (2): 55-121.

5. Dias PC, Henriques P, Anjos, Luiz Ant., Burlandy L. Obesidade e Políticas Públicas: Concepções e Estratégias Adotadas Pelo Governo Brasileiro. Cad. Saúde Pública. 2017; 17 (33): 1678-4464.

6. Nascimento JF, Silva DRF, Barbosa CHCB, Santos FV, Martins M, Rodrigues PLDC. Sobrepeso e obesidade em adolescentes escolares: uma revisão sistemática. Saúde Coletiva. 2020; 10(55), 2947-958.

7. Novelle JM, Alvarenga MS. Cirurgia bariátrica e transtornos alimentares: uma revisão integrativa. J. bras. psiquiatr. 2016; 65(3): 33-41.

8. Nunes PAN, Valadão JA, Malafaia O, Martins OJT, Garcia RF, Klostemann FC. Modificação técnica para a gastrectomia vertical. ABCD, arq. bras. cir. dig. 2018; 26(1): 74-80.

9. Costa R., Carvalho LRA, Lima MD, Costa TPC, Onyeisi JS Avaliação do consumo de medicamentos para o tratamento da obesidade: um estudo realizado em farmácias do município de Teresina-Piauí. Research, Society and Development. 2020; 9(3): 293-310, set. 2020.

10. Carlos Neto, D. C.; Souza, A. P. B.; Pilonetto, R. L. O Uso Off Label de Psicotrópicos no Tratamento da Obesidade. Revista Científica Multidisciplinar Núcleo do Conhecimento. 2016(16):308320.

11. Martins JS, Moura MBS, Brito MHRM. Avaliação do consumo de medicamentos emagrecedores dispensado em uma drogaria. Research, Society and Development. 2020; 9(6): 789-95.
12. Luz F, Hay P, Stephen Touyz S, Sainsbury A. Affiliations Obesity with Comorbid Eating Disorders: Associated Health Risks and Treatment Approaches. Nutrientes. 2018; 10 (7): 829.

13. Kalaria SN, McElroy SL, Gobburu J, Gopalakrishnan M. An Innovative Disease-Drug-Trial Framework to Guide Binge Eating Disorder Drug Development: A Case Study for Topiramato. Clin Transl Sci. 2019; 10(1): 1-10.

14. Wang C, Shi W, Xu J, Huang C, Zhu J. Outcomes and safety of concomitant topiramate or metformin for antipsychotics-induced obesity: a randomized-controlled trial. Ann Gen Psychiatry. 2020; 10;19(1):68.

15. Sari C, Seip RL, Umashanker D. Case Report: Off Label Utilization of Topiramate and Metformin in Patients With BMI $\geq 50$ $\mathrm{kg} / \mathrm{m} 2$ Prior to Bariatric Surgery. Case Report: Pharmacological Weight Loss With Generics. 2021; 12(1): 1-6.

16. Kolotkin RL, Gadde KM, Peterson CA, Crosby RD. Health-related quality of life in two randomized controlled trials of phentermine/topiramate for obesity: What mediates improvement? Qual Life Res. 2016;25(5):1237-244.

17. Hsia DS, Gosselin NH, Williams J, Farhat N, Marier JF, Shih W, Peterson C, Siegel R. A randomized, double-blind, placebo-controlled, pharmacokinetic and pharmacodynamic study of a fixeddose combination of phentermine/topiramate in adolescents with obesity. Diabetes Obes Metab. 2020; 22(4):480-91.

18. Istfan NW, Anderson WA, Hess DT, Yu L, Carmine B, Apovian CM. The Mitigating Effect of Phentermine and Topiramate on Weight Regain After Roux-en-Y Gastric Bypass Surgery. Obesity (Silver Spring). 2020; 28(6):1023-030.

19. Lei XG, Lai C, Yang X. Efficacy and safety of phentermine/ topiramate in adults with overweight or obesity: A meta-analysis and systematic review. MedRxiv preprint. 2020; 12(1): 1-25.

20. Safer DL, Adler S, Dalai SS, Jason PMS, Toyama BH, Najarian SPT. A randomized, placebo controlled crossover trial of phentermine topiramate ER in patients with binge eating disorder and bulimia nervosa. First published. 2019; 53(2): 13-21.

21. Halpern B, Mancini MC. Safety assessment of combination therapies in the treatment of obesity: focus on naltrexone/bupropion extended release and phentermine-topiramate extended release. Expert opin drug saf. 2017; 16(21): 27-39.

22. Nithyakala P, Sathyaprabha G, Venila J. Study on Efficacy of Topiramate: Impact on weight Loss in Obese patients. Expert opin drug saf. $2021 ; 14(3)$ : 21-35. 